\title{
Caracterización sociodemográfica y clínica de una población con falla cardíaca aguda: cohorte MED-ICA
}

\section{Oscar A. Muñoz-Mejía ${ }^{\mathrm{a}, \mathrm{b}, \mathrm{e}, *}$, Elsy C. Sierra-Vargas ${ }^{\mathrm{c}, \mathrm{b}, \mathrm{e}}$, Andrés Zapata-Cárdenas ${ }^{\mathrm{d}, \mathrm{c}, \mathrm{e}}$, Mariana Isaza-Montoya ${ }^{e}$, María A. Muñoz-Cifuentes ${ }^{e}$, Juan D. Sánchez-Echavarría ${ }^{e}$ y Javier Echeverri-García ${ }^{e}$, en nombre del Grupo de Investigación en Medicina Interna (GIMI), Facultad de Medicina, Universidad de Antioquia-IPS Universitaria}

\footnotetext{
a Clínica Cardiovascular Santa María, CardioVID, Medellín, Colombia

b Universidad Cooperativa de Colombia, Medellín, Colombia

c Hospital Universitario San Vicente Fundación, Medellín, Colombia

d IPS Universitaria León XIII, Medellín, Colombia

e Universidad de Antioquia, Medellín, Colombia
}

Recibido el 19 de octubre de 2017; aceptado el 5 de diciembre de 2017

Disponible en Internet el 10 de mayo de 2018

\section{PALABRAS CLAVE \\ Insuficiencia cardiaca aguda; \\ Mortalidad; \\ Factores de riesgo}

\begin{abstract}
Resumen
Objetivo: Determinar las características epidemiológicas de adultos con insuficiencia cardiaca aguda admitidos en un hospital universitario.

Métodos: Estudio de cohorte retrospectivo, descriptivo. Revisión de historias clínicas de mayores de 18 años hospitalizados entre julio y diciembre de 2015 en un hospital de Medellín, Colombia.

Resultados: Se incluyeron 361 pacientes con insuficiencia cardíaca aguda. 193 (53,4\%) fueron mujeres, cuya mediana de edad fue 76 años. 183 (50,6\%) tenían fracción de eyección (FEVI) reducida, 19 (5,2\%) FEVI intermedia y 148 (40,9\%) FEVI preservada. El tratamiento farmacológico previo al ingreso incluía IECA/ARA2 en 253 (70\%) pacientes, betabloqueador en $212(58,7 \%)$ y espironolactona en $92(25,4 \%)$. La principal causa de falla cardíaca aguda fue la presencia de taqui-bradiarritmias $(17,5 \%)$, seguida de infección $(17,2 \%)$ y exacerbación de neumopatía crónica (16,3\%). La clasificación clínica de la descompensación fue Stevenson B en 335 (92,7\%) pacientes, Stevenson C en $20(5,5 \%)$ y Stevenson L en 6 (1,6\%). La mediana de hospitalización fue 6 días (4-9 días). 30 pacientes $(8,3 \%$ ) fallecieron, $50 \%$ por infecciones asociadas a la descompensación cardiaca y $20 \%$ por síndrome coronario agudo.
\end{abstract}

\footnotetext{
* Autor para correspondencia.

Correo electrónico: oscarmu@hotmail.com (O.A. Muñoz-Mejía).
} 
Conclusión: Se hallaron similitudes con estudios internacionales, pero mayor mortalidad vinculada principalmente a infección como factor precipitante de descompensación. Se caracterizaron factores desencadenantes y etiología, datos útiles en la práctica clínica. Hubo alta carga de comorbilidades, cuya descompensación impactó de manera significativa en la agudización de la falla cardiaca. El subgrupo con fracción de eyección intermedia presentó particularidades que ameritan mayor caracterización.

(c) 2018 Sociedad Colombiana de Cardiología y Cirugía Cardiovascular. Publicado por Elsevier España, S.L.U. Este es un artículo Open Access bajo la licencia CC BY-NC-ND (http:// creativecommons.org/licenses/by-nc-nd/4.0/).

\section{KEYWORDS}

Acute heart failure;

Mortality;

Risk factors

\section{Sociodemographic and clinical profile of a population with acute heart failure: MED-ICA cohort}

\begin{abstract}
Objective: To determine the epidemiological characteristics of adults with acute heart failure admitted to a University Hospital.

Method: A retrospective, descriptive cohort study conducted by reviewing the medical notes of patients over 18 years-old and admitted between July and December 2015 to a hospital in Medellin, Colombia.

Results: The study included 361 patients with acute heart failure, with a mean age of 76 years, and of whom 193 (53.4\%) were women. A reduced ejection fraction (LVEF) was observed in 183 (50.6\%) patients, 19 (5.2\%) with an intermediate LVEF, and 148 (40.9\%) with normal LEVF. The pharmacological treatment prior to admission included angiotensin-converting-enzyme (ACE) inhibitors / angiotensin II receptor antagonists (ARA2) in 253 (70\%) patients, a beta-blocker in $212(58.7 \%)$, and spironolactone in $92(25.4 \%)$. The main cause of acute heart failure was the presence of tachy-brady-arrhythmias (17.5\%), followed by infection (17.2\%), and exacerbation of chronic pulmonary disease (16.3\%). The clinical classification of the decompensation was Stevenson B in $335(92.7 \%)$ patients, Stevenson C in 20 (5.5\%), and Stevenson L in $6(1.6 \%)$. The mean admission time was 6 (4-9) days. There were $30(8.3 \%)$ deaths, $50 \%$ due to infections associated with cardiac decompensation and $20 \%$ due to acute coronary syndrome.

Conclusion: Similarities were found with international studies, but there was a higher mortality mainly linked to infection as a decompensation precipitating factor. The triggering factors and aetiology are presented, which are useful data in clinical practice. There was a high level of comorbidities, and their decompensation had a significant impact on the exacerbation of heart failure. The sub-group with the intermediate ejection fraction had features that require further characterisation.

(C) 2018 Sociedad Colombiana de Cardiología y Cirugía Cardiovascular. Published by Elsevier España, S.L.U. This is an open access article under the CC BY-NC-ND license (http:// creativecommons.org/licenses/by-nc-nd/4.0/).
\end{abstract}

\section{Introducción}

La insuficiencia cardiaca aguda es un motivo de consulta frecuente en los servicios de urgencias. Es la principal causa de hospitalización en mayores de 65 años, con tasas de mortalidad intrahospitalaria entre el 4 y el $10 \%^{1-3}$ y de hasta un $50 \%$ a un año ${ }^{4}$. El término insuficiencia cardíaca aguda describe el inicio o la recurrencia de signos y síntomas de falla cardiaca que requieren terapia urgente o emergente, y que resultan en la necesidad de cuidado médico imprevisto o de hospitalización. Aunque el término "agudo" sugiere un inicio súbito de síntomas, muchos pacientes pueden tener un curso más subagudo, con empeoramiento gradual de síntomas que finalmente alcanzan un nivel de gravedad suficiente que los lleva a consultar ${ }^{5,6}$. Representa un reto diagnóstico y terapéutico, especialmente en presencia de comorbilidades donde los signos y síntomas pueden sobreponerse.

En Colombia son pocos los estudios que describen el comportamiento clínico y epidemiológico de los pacientes con falla cardiaca aguda ${ }^{7-11}$. Consideramos pertinente caracterizar nuestra propia población. El conocimiento de los factores precipitantes de descompensación puede contribuir al desarrollo de estrategias de prevención y a la optimización del manejo en estos pacientes.

Los objetivos principales de este estudio son los siguientes:

- Determinar las características epidemiológicas de los pacientes con diagnóstico de insuficiencia cardiaca aguda.

- Evaluar las diferencias en el perfil clínico de acuerdo con el género, la fracción de eyección y el perfil hemodinámico.

- Establecer la frecuencia de mortalidad intrahospitalaria.

\section{Métodos}

Se realizó un estudio de cohorte retrospectivo, con registros clínicos de la IPS universitaria León XIII de la ciudad de 
Medellín, Colombia. Se revisaron historias clínicas con código diagnóstico CIE-10 para insuficiencia cardíaca (I50X) entre los meses de julio y diciembre del año 2015. Se incluyeron pacientes mayores de 18 años con diagnóstico principal de insuficiencia cardiaca aguda por criterios de Framingham (dos criterios mayores o un criterio mayor y dos criterios menores) $)^{12}$. Se excluyeron embarazadas, a quienes no requirieron hospitalización y aquellos en quienes posterior a la revisión de la historia clínica se concluyó que no tenían insuficiencia cardiaca aguda. Se utilizó un formato de recolección electrónico diseñado por el grupo GIMI. Las dudas que surgieron del análisis fueron corroboradas con una nueva revisión de la historia clínica.

Se estudiaron como variables: género, edad, zona geográfica de procedencia, antecedentes patológicos (enfermedad coronaria, hipertensión arterial, fibrilación auricular, diabetes mellitus, enfermedad renal crónica, enfermedad pulmonar obstructiva crónica, entre otros), antecedentes farmacológicos (diuréticos, inhibidores de la enzima convertidora de angiotensina -IECA-, antagonistas de receptores de angiotensina II -ARAll-, espironolactona, digoxina), uso de dispositivos cardiacos, características de la insuficiencia cardiaca establecida (tiempo de evolución desde el diagnóstico, clasificación funcional New York Heart Association (NYHA) basal (previo al inicio de signos y síntomas de falla cardiaca) y al ingreso hospitalario. La fracción de eyección del ventrículo izquierdo se obtuvo a partir del ecocardiograma realizado por el servicio de cardiología institucional o de estudio previo no mayor a tres meses, y fue definida de la siguiente manera: reducida o $\leq 40 \%$, preservada o $\geq 40 \%$ y adicionalmente se analizó el subgrupo con fracción de eyección intermedia si ésta se encontraba entre 41 y $49 \%$.

Se describieron las características del episodio clínico actual: tiempo de evolución de los síntomas, manifestaciones clínicas de insuficiencia cardiaca aguda, signos vitales al ingreso a urgencias, perfil hemodinámico de Stevenson al ingreso, uso de soporte vasoactivo durante la hospitalización (inotrópicos, vasopresores o vasodilatadores), días de hospitalización, estancia en unidad de cuidados intensivos (UCl) y mortalidad intrahospitalaria.

Se determinó la causa de la descompensación y se clasificó en los siguientes grupos: asociado a aparición de bradiarritmias o taquiarritmias, síndrome coronario agudo, infección, exacerbación de comorbilidades de base (enfermedad pulmonar obstructiva crónica, diabetes, hipertensión arterial no controlada, lesión renal crónica agudizada, anemia), no adherencia a manejo farmacológico, otra causa y causa no aclarada.

La etiología de la insuficiencia cardiaca fue clasificada en seis grupos: hipertensiva, isquémica, valvular, arritmia, otra etiología (miocarditis, tóxica, asociada a alteración tiroidea, asociada a colagenopatía, entre otros), y etiología no aclarada (idiopática).

Las variables de laboratorio registradas al ingreso fueron: hemoglobina $(\mathrm{g} / \mathrm{dl})$, creatinina $(\mathrm{mg} / \mathrm{dl})$ y nitrógeno ureico en sangre $(\mathrm{mg} / \mathrm{dl})$, electrolitos séricos (potasio, sodio y cloro en $\mathrm{mmol} / \mathrm{L})$, troponina l $(\mathrm{pg} / \mathrm{ml})$ y tirotropina $(\mathrm{mU} / \mathrm{L})$.

Las variables continuas se presentan como medias aritméticas y medianas con rango intercuartílico (RIC), y las variables categóricas se presentan como frecuencias y proporciones.
El proyecto de investigación fue aprobado, previo a su ejecución, por el comité de ética de la IPS universitaria Clínica León XIII. El estudio fue financiado en su totalidad por los autores.

\section{Resultados}

\section{Características demográficas y clínicas}

Las tablas 1 y 2 presentan las características demográficas y clínicas más importantes, distribuidas según la fracción de eyección. Entre el 1 de julio y 31 de diciembre de 2015 se presentaron al servicio de urgencias 361 adultos con insuficiencia cardiaca aguda, 193 mujeres $(53,4 \%)$ y 168 hombres $(46,6 \%)$. La mediana de edad fue 76 años (RIC 65 - 83 años), discretamente mayor en mujeres (mediana 79 vs. 73 años). Las comorbilidades más comunes fueron: hipertensión arterial $(86,1 \%)$, neumopatía crónica tipo enfermedad pulmonar obstructiva crónica $(44,3 \%)$, diabetes mellitus $(30,7 \%)$, dislipidemia $(26,8 \%)$, fibrilación auricular $(26,3 \%)$, enfermedad renal crónica $(24,4 \%)$ y enfermedad tiroidea $(20,8 \%)$. Cerca de una cuarta parte $(23,8 \%)$ de los pacientes tenía antecedente de enfermedad coronaria. Los medicamentos usados con mayor frecuencia como tratamiento de base fueron diuréticos de asa $(60,3 \%)$, betabloqueadores $(58,7 \%)$, ARA2 $(40,1 \%)$ e IECA $(29,9 \%)$.

Cien individuos $(27,7 \%)$ presentaron insuficiencia cardíaca de novo. De los 261 pacientes con antecedente conocido de insuficiencia cardiaca, al menos $148(56,7 \%)$ habían tenido, como mínimo, un episodio previo de falla cardíaca descompensada. El tiempo de evolución de los síntomas de insuficiencia cardíaca tuvo una mediana de 7 días (RIC 3 - 15). El principal síntoma de descompensación fue la disnea $(90,3 \%)$, seguido de edema periférico $(81,9 \%)$ y ortopnea $(46,8 \%)$. Los signos clínicos de agudización de falla cardiaca descritos con mayor frecuencia fueron crépitos pulmonares $(42,1 \%)$ y distensión yugular $(22,9 \%)$.

Se evaluó la clasificación funcional NYHA basal y al ingreso. Solo el $9 \%$ de los pacientes tenían NYHA clase IV basal, y como era de esperarse, este porcentaje aumentó al $67 \%$ al momento del ingreso hospitalario. Hasta el $96 \%$ de individuos se presentó con NYHA clase III o IV al ingreso.

Se obtuvieron 183 pacientes $(50,6 \%)$ con fracción de eyección reducida y 167 individuos $(46,2 \%)$ con fracción de eyección preservada; de éstos, 19 pacientes $(5,2 \%$ del total de la muestra) presentaron fracción de eyección intermedia. La mediana de edad en el grupo con fracción de eyección <40\% fue 74 años (RIC 65 - 81), comparado con 77 años (RIC 67 - 84) en pacientes con fracción de eyección mayor de 40\%. Los pacientes con fracción de eyección intermedia tuvieron la mediana de edad más alta (81 años, RIC 76 - 88). El 80,3\% de los pacientes con fracción de eyección reducida y el $65,2 \%$ de los pacientes con fracción de eyección preservada tenían diagnóstico previo de insuficiencia cardíaca. Por lo menos el $51,9 \%$ de los pacientes con FEVI reducida habían tenido mínimo un episodio previo de descompensación, en comparación con los pacientes con FEVI preservada, en quienes la frecuencia fue de solo $30,1 \%$. En el grupo con FEVI preservada predominó el antecedente de fibrilación auricular (32,9\% vs. $20,7 \%)$ y de trastornos tiroideos $(24,5 \%$ vs. 16,3\%); En cambio, en el grupo con FEVI 
Tabla 1 Antecedentes y características demográficas según la FEVI

\begin{tabular}{|c|c|c|c|}
\hline \multirow[t]{2}{*}{ Variable } & \multirow{2}{*}{$\begin{array}{l}\text { Total } \\
361(100 \%)\end{array}$} & \multirow{2}{*}{$\begin{array}{l}\text { FEVI }<40 \% \\
183(50,6 \%)\end{array}$} & \multirow{2}{*}{$\begin{array}{l}\text { FEVI }>40 \% \\
167(46,2 \%) \\
\end{array}$} \\
\hline & & & \\
\hline Edad (mediana en años) & $76(65-83)$ & $74(65-81)$ & $77(67-84)$ \\
\hline Género femenino (\%) & $193(53,4)$ & $80(43,7)$ & $107(64,1)$ \\
\hline Procedencia urbana (\%) & $323(89,5)$ & $163(89,0)$ & $150(89,8)$ \\
\hline \multicolumn{4}{|l|}{ Tratamiento previo a la descompensación } \\
\hline Diuréticos de asa (\%) & $218(60,3)$ & $124(66,3)$ & $88(52,6)$ \\
\hline Betabloqueadores (\%) & $212(58,7)$ & $121(64,7)$ & $86(51,5)$ \\
\hline ARA2 (\%) & $145(40,1)$ & $74(39,5)$ & $69(41,3)$ \\
\hline IECA (\%) & $108(29,9)$ & $67(35,8)$ & $39(23,3)$ \\
\hline Espironolactona (\%) & $92(25,4)$ & $68(36,3)$ & $22(13,1)$ \\
\hline Calcioantagonistas (\%) & $84(23,2)$ & $30(16,0)$ & $53(31,7)$ \\
\hline Diuréticos tiazídicos (\%) & $20(5,5)$ & $3(1,6)$ & $15(8,9)$ \\
\hline Digitálicos (\%) & $15(4,1)$ & $14(7,4)$ & $1(0,6)$ \\
\hline Estatinas (\%) & $176(48,7)$ & $95(50,8)$ & $78(46,7)$ \\
\hline Antiplaquetarios (\%) & $131(36,2)$ & $75(40,1)$ & $53(31,7)$ \\
\hline Anticoagulantes (\%) & $78(21,6)$ & $41(66,3)$ & $36(21,5)$ \\
\hline \multicolumn{4}{|l|}{ Uso de dispositivos cardiacos } \\
\hline Marcapasos (\%) & $31(8,4)$ & $16(8,7)$ & $13(7,7)$ \\
\hline Cardiodesfibrilador (\%) & $5(1,3)$ & $3(1,6)$ & $1(0,6)$ \\
\hline Resincronizador cardiaco (\%) & $2(0,5)$ & $2(1,0)$ & $0(0,0)$ \\
\hline \multicolumn{4}{|l|}{ Principales comorbilidades } \\
\hline Hipertensión arterial (\%) & $311(86,1)$ & $153(83,6)$ & $149(89,2)$ \\
\hline Neumopatía crónica (EPOC, asma, EPID) (\%) & $160(44,3)$ & $81(44,2)$ & $72(43,1)$ \\
\hline Diabetes mellitus (\%) & $111(30,7)$ & $60(32,7)$ & $48(28,7)$ \\
\hline Dislipidemia (\%) & $97(26,8)$ & $52(28,4)$ & $42(25,2)$ \\
\hline Fibrilación auricular (\%) & $95(26,3)$ & $38(20,7)$ & $55(32,9)$ \\
\hline ERC estadio 3 o superior (\%) & $88(24,3)$ & $44(24,0)$ & $42(25,1)$ \\
\hline Enfermedad coronaria (\%) & $86(23,8)$ & $54(29,5)$ & $29(17,3)$ \\
\hline Enfermedad tiroidea (\%) & $75(20,7)$ & $30(16,3)$ & $41(24,5)$ \\
\hline Enfermedad cerebrovascular (\%) & $36(9,9)$ & $23(12,5)$ & $12(7,1)$ \\
\hline Cáncer (\%) & $35(9,7)$ & $14(7,6)$ & $19(11,3)$ \\
\hline $\operatorname{Otros}^{\mathrm{a}}(\%)$ & $70(19,4)$ & $34(18,6)$ & $32(19,2)$ \\
\hline
\end{tabular}

FEVI: fracción de eyección del ventrículo izquierdo; IECA: inhibidores de la enzima convertidora de angiotensina; ARA2: antagonistas de receptor de angiotensina 2, EPOC: enfermedad pulmonar obstructiva crónica, EPID: enfermedad pulmonar intersticial difusa.

a Anemia (25), tabaquismo (23), hepatopatías (9), hipertensión pulmonar (6), enfermedades neurodegenerativas (5), VIH (2).

reducida se documentó mayor frecuencia de enfermedad coronaria $(29,5 \%$ vs. $17,3 \%)$. En cuanto al tratamiento de base, aquellos con FEVI reducida, en comparación con los pacientes de FEVI preservada, usaban con mayor frecuencia diuréticos de asa $(67,2 \%$ vs. $52,6 \%)$, beta-bloqueadores $(65 \%$ vs. $51,5 \%)$, IECA $(35,5 \%$ vs. $23,3 \%)$, espironolactona $(37,1 \%$ vs. $13,1 \%)$ y digitálicos $(7,6 \%$ vs. $0,6 \%$ ). Por su parte, aquellos con FEVI preservada utilizaban más calcioantagonistas $(31,7 \%$ vs. $15,3 \%)$ y diuréticos tiazídicos $(8,9 \%$ vs. $1,6 \%)$ en comparación con el grupo de pacientes con $\mathrm{FEVI}$ reducida. $\mathrm{El}$ tiempo de evolución de manifestaciones clínicas fue similar en ambos grupos. No hubo diferencias importantes en la frecuencia de aparición de los síntomas entre estos dos grupos; sin embargo, el número de individuos con descompensación Stevenson $\mathrm{C}$ fue mayor en el grupo con FEVI reducida vs. preservada $(8,2 \%$ vs. $2,4 \%)$.

Respecto al perfil hemodinámico de descompensación al ingreso, predominó la clasificación Stevenson B (92,7\%), seguida por la clasificación Stevenson C $(5,5 \%)$ y Stevenson
L (1,6\%). Aquellos clasificados Stevenson B fueron ligeramente mayores que el grupo Stevenson $C$ y $L$ (mediana 76 vs. 72,5 años). Los pacientes Stevenson B fueron en su mayoría mujeres $(53,7 \%)$ pero en el grupo de pacientes $C$ o $\mathrm{L}$ la proporción de hombres y mujeres fue similar. Por lo menos el $46 \%$ de los pacientes Stevenson C o $L$ habían tenido como mínimo un episodio previo de descompensación, pero en el grupo Stevenson este antecedente fue un poco menos frecuente $(40,6 \%$ de casos). Se observó que el uso previo de diuréticos tiazídicos, IECA, ARA2, calcioantagonistas y estatinas fue mayor en el grupo Stevenson B. El uso previo de otros medicamentos, la utilización de dispositivos cardíacos, las comorbilidades y las constantes vitales al ingreso fueron, en general, similares para los tres grupos. La mediana del tiempo de evolución del cuadro clínico de la descompensación fue menor en los pacientes Stevenson C o L (4,5 días vs. 7 días). Algunos síntomas típicos fueron más frecuentes en el grupo Stevenson B, que en aquellos Stevenson C o L (ortopnea 48,9\% 
Tabla 2 Características clínicas al ingreso según la FEVI

\begin{tabular}{|c|c|c|c|}
\hline \multirow[t]{2}{*}{ Variable } & Total & $\mathrm{FEVI}<40 \%$ & $\mathrm{FEVI}>40 \%$ \\
\hline & $361(100 \%)$ & $183(50,6 \%)$ & $167(46,2 \%)$ \\
\hline \multicolumn{4}{|l|}{ Presentación clínica de la descompensación } \\
\hline Al menos un episodio previo de descompensación (\%) & $148(41,0)$ & $95(51,1)$ & $50(30,1)$ \\
\hline Evolución en días de la descompensación (\%) & $7(3-15)$ & $8(3-15)$ & $7(3-15)$ \\
\hline Disnea (\%) & $326(90,3)$ & $163(89,0)$ & $153(91,6)$ \\
\hline Edema periférico (\%) & $296(81,9)$ & $153(83,6)$ & $138(82,6)$ \\
\hline Ortopnea (\%) & $169(46,8)$ & $91(49,7)$ & $76(45,5)$ \\
\hline Disnea paroxística nocturna (\%) & $123(34,0)$ & $66(36,0)$ & $56(33,5)$ \\
\hline Crépitos pulmonares (\%) & $152(42,1)$ & $70(38,2)$ & $74(44,3)$ \\
\hline Ingurgitación yugular (\%) & $83(22,9)$ & $48(26,2)$ & $33(19,7)$ \\
\hline Dolor torácico $(\%)$ & $64(17,3)$ & $35(19,1)$ & $27(16,1)$ \\
\hline Soplo cardíaco (\%) & $30(8,3)$ & $18(9,8)$ & $10(5,9)$ \\
\hline Fiebre (\%) & $28(7,7)$ & $11(6,0)$ & $16(9,5)$ \\
\hline Tercer ruido (\%) & $7(1,9)$ & $3(1,6)$ & $4(2,4)$ \\
\hline Reflujo hepatoyugular (\%) & $10(2,7)$ & $6(3,2)$ & $4(2,4)$ \\
\hline Tos (\%) & $61(16,9)$ & $29(15,8)$ & $29(17,3)$ \\
\hline Otro $^{\mathrm{a}}(\%)$ & $84(23,2)$ & $41(22,4)$ & $39(23,3)$ \\
\hline \multicolumn{4}{|l|}{ NYHA de base } \\
\hline NYHA I (\%) & $24(6,6)$ & $12(6,5)$ & $10(5,9)$ \\
\hline NYHA II (\%) & $149(41,2)$ & $74(40,4)$ & $72(43,1)$ \\
\hline NYHA III (\%) & $131(35,2)$ & $68(37,1)$ & $61(36,5)$ \\
\hline NYHA IV (\%) & $33(9,1)$ & $17(9,2)$ & $13(7,7)$ \\
\hline Desconocida (\%) & $24(6,6)$ & $12(6,5)$ & $11(6,5)$ \\
\hline \multicolumn{4}{|l|}{ NYHA al ingreso } \\
\hline NYHA II (\%) & $16(4,4)$ & $6(3,2)$ & $9(5,3)$ \\
\hline NYHA III (\%) & $103(28,5)$ & $52(28,4)$ & $50(29,9)$ \\
\hline NYHA IV (\%) & $241(66,7)$ & $124(67,7)$ & $108(64,6)$ \\
\hline \multicolumn{4}{|l|}{ Perfil hemodinámico (Stevenson) } \\
\hline Stevenson B (\%) & $335(92,8)$ & $166(90,7)$ & $160(95,8)$ \\
\hline Stevenson C (\%) & $20(5,5)$ & $15(8,2)$ & $4(2,4)$ \\
\hline Stevenson L (\%) & $6(1,6)$ & $2(1,0)$ & $3(1,8)$ \\
\hline
\end{tabular}

FEVI: fracción de eyección del ventrículo izquierdo; NYHA: New York Heart Association.

a Dolor abdominal, astenia, cianosis, alteración del sensorio, vómito, mareo y diarrea.

Tabla 3 Características de laboratorio según la FEVI*

\begin{tabular}{llll}
\hline Variable & Total $^{*}$ & FEVI<40\% & FEVI >40\% \\
\cline { 2 - 4 } & $361(100 \%)$ & $183(50,7 \%)$ & $167(46,3 \%)$ \\
\hline Potasio $(\mathrm{mmol} / \mathrm{L})(\mathrm{n}=346)$ & $4,3(3,9-4,73)$ & 4,3 & 4,3 \\
Sodio $(\mathrm{mmol} / \mathrm{L})(\mathrm{n}=346)$ & $140,2(138-143)$ & 140,2 & 140,2 \\
Cloro $(\mathrm{mmol} / \mathrm{L})(\mathrm{n}=341)$ & $105(101,3-108,2)$ & 105 & 24,05 \\
Nitrógeno ureico $(\mathrm{mg} / \mathrm{dl})(\mathrm{n}=354)$ & $24,05(17,6-34,5)$ & 1,05 \\
Creatinina $(\mathrm{mg} / \mathrm{dl})(\mathrm{n}=360)$ & $1,05(0,82-1,46)$ & 12,7 \\
Hemoglobina $(\mathrm{g} / \mathrm{dl})(\mathrm{n}=361)$ & $12,7(11,2-14,5)$ & 1,05 & 23 \\
Troponina I pg/ml $(\mathrm{m}=200)$ & $23(4,8-51,3)$ & 12,7 & 2,7 \\
TSH mU/L $(\mathrm{n}=102)$ & $2,7(1,39-6,9)$ & 23 & 2,65 \\
\hline
\end{tabular}

\footnotetext{
* Los resultados se expresan en mediana.

a Rango de referencia del laboratorio: $0,0-34,1 \mathrm{pg} / \mathrm{ml}$.
} 
Tabla 4 Etiología y factores desencadenantes asociados a insuficiencia cardíaca aguda

\begin{tabular}{|c|c|c|c|c|c|c|}
\hline \multirow[t]{2}{*}{ Variable } & Total & $\mathrm{FEVI}<40 \%$ & $\mathrm{FEVI}>40 \%$ & Stevenson B & Stevenson C & Stevenson L \\
\hline & $361(100 \%)$ & $183(50,7 \%)$ & $167(46,3 \%)$ & $335(92,8 \%)$ & $20(5,5 \%)$ & $6(1,4 \%)$ \\
\hline \multicolumn{7}{|l|}{ Etiología de la insuficiencia cardíaca } \\
\hline Hipertensiva & $167(46,3 \%)$ & $81(44,3 \%)$ & $81(48,5 \%)$ & $158(47,2 \%)$ & $7(35 \%)$ & $2(33,3 \%)$ \\
\hline Isquémica & $120(33,2 \%)$ & $85(46,5 \%)$ & $33(19,8 \%)$ & $110(32,8 \%)$ & $8(40 \%)$ & $2(33,3 \%)$ \\
\hline Valvular & $42(11,6 \%)$ & $23(12,6 \%)$ & $18(10,8 \%)$ & $38(11,3 \%)$ & $3(15 \%)$ & $1(13,7 \%)$ \\
\hline Arritmia & $35(9,7 \%)$ & $16(8,7 \%)$ & $18(10,8 \%)$ & $29(8,7 \%)$ & $5(25 \%)$ & 0 \\
\hline Idiopática & $28(7,8 \%)$ & $8(4,3 \%)$ & $19(11,3 \%)$ & $28(8,3 \%)$ & 0 & $1(13,7 \%)$ \\
\hline Otras* & $15(4,2 \%)$ & $9(4,9 \%)$ & $6(3,6 \%)$ & $15(4,5 \%)$ & 0 & 0 \\
\hline Más de dos etiologías & $72(19,9 \%)$ & $46(25,1 \%)$ & $24(14,3 \%)$ & $68(20,2 \%)$ & $3(15 \%)$ & $1(13,7 \%)$ \\
\hline \multicolumn{7}{|l|}{ Factor desencadenante } \\
\hline Taquiarritmia o bradiarritmia & $63(17,5 \%)$ & $36(19,7 \%)$ & $27(16,2 \%)$ & $58(17,3 \%)$ & $4(20 \%)$ & $1(13,7 \%)$ \\
\hline Infección & $62(17,2 \%)$ & $27(14,7 \%)$ & $34(20,4 \%)$ & $56(16,7 \%)$ & $5(25 \%)$ & $1(13,7 \%)$ \\
\hline Exacerbación neumopatía crónica & $59(16,3 \%)$ & $23(12,6 \%)$ & $31(18,6 \%)$ & $55(16,4 \%)$ & $3(15 \%)$ & $1(13,7 \%)$ \\
\hline No adherencia al tratamiento & $50(13,9 \%)$ & $34(18,6 \%)$ & $16(9,6 \%)$ & $46(13,7 \%)$ & $3(15 \%)$ & $1(13,7 \%)$ \\
\hline Hipertensión no controlada & $48(13,3 \%)$ & $26(14,2 \%)$ & $20(12 \%)$ & $44(13,1 \%)$ & $2(10 \%)$ & $2(33,3 \%)$ \\
\hline Disfunción renal aguda & $31(8.6 \%)$ & $16(8,7 \%)$ & $15(9 \%)$ & $31(9,3 \%)$ & 0 & 0 \\
\hline Síndrome coronario agudo & $26(7,2 \%)$ & $16(8,7 \%)$ & $9(5,4 \%)$ & $25(7,5 \%)$ & $1(5 \%)$ & 0 \\
\hline Anemia & $14(3,9 \%)$ & $3(1,6 \%)$ & $10(6 \%)$ & $14(4,2 \%)$ & 0 & 0 \\
\hline Descompensación DM & $13(3,6 \%)$ & $6(3,3 \%)$ & $6(3,6 \%)$ & $12(3,6 \%)$ & $1(5 \%)$ & 0 \\
\hline Causa no clara & $55(15,2 \%)$ & $26(14,2 \%)$ & $20(12 \%)$ & $45(13,4 \%)$ & $1(5 \%)$ & $2(33,3 \%)$ \\
\hline Otro $^{a}$ & $21(5,8 \%)$ & $12(6,6 \%)$ & $9(5,4 \%)$ & $18(5,4 \%)$ & $3(15 \%)$ & 0 \\
\hline Más de dos factores desencadenantes & $72(19,9 \%)$ & $41(22,4 \%)$ & $29(17,4 \%)$ & $66(19,7 \%)$ & $4(20 \%)$ & $2(33,3 \%)$ \\
\hline
\end{tabular}

"Miocarditis (5), tóxica (4), anemia (3), TEP (3), mixedema tiroideo (1), SAHOS (1), cardiopatía lúpica (1).

a Disfunción tiroidea (7), iatrogenia (7), pancreatitis aguda (3), disfunción dispositivo cardiaco (2), TEP (2).

vs. $19,2 \%$ ) y disnea paroxística nocturna $35,8 \%$ vs. $11,5 \%)$. Por otra parte, en los pacientes Stevenson C o L predominó la presencia de síntomas no típicos de ICA (50\% vs. $21,1 \%$ ); estos síntomas comprendían dolor abdominal, astenia y adinamia, cianosis, alteraciones del nivel de consciencia, vómito, mareo y diarrea.

\section{Hallazgos de laboratorio}

La tabla 3 muestra las características de laboratorio de la cohorte clasificadas según la fracción de eyección. No se observaron diferencias importantes en los niveles de electrolitos, hemoglobina, azoados, troponina I, ni tirotropina al ingreso. No se encontraron diferencias importantes en los laboratorios según la clasificación de Stevenson o en el grupo de individuos fallecidos.

\section{Etiología de la falla cardiaca y causas de descompensación}

La tabla 4 presenta la etiología de la insuficiencia cardiaca y los factores desencadenantes de descompensación. La principal etiología de falla cardiaca fue la hipertensiva $(46,3 \%)$, seguida de la isquémica $(33,2 \%)$, valvular $(11,6 \%)$ y arrítmica $(9,7 \%)$. No se documentó etiología de la falla cardiaca en el $7,8 \%$ de los pacientes. En el grupo con FEVI reducida predominó la etiología isquémica $(46,5 \%)$, en contraste con aquellos de FEVI preservada, en quienes predominó la etiología hipertensiva $(48,5 \%)$. Se documentó etiología mixta
( 2 o más causas de falla cardiaca) en 72 individuos (19,9\%) y fue más frecuente en el grupo con FEVI reducida que en el de FEVI preservada $(25,1 \%$ vs. $14,3 \%)$. La etiología hipertensiva fue la que más se asoció con falla cardiaca de origen mixto, coexistiendo en 61 pacientes $(84,7 \%$ de los pacientes con etiología mixta).

Al evaluar la etiología según el perfil hemodinámico de Stevenson se encontró que en los pacientes Stevenson B predominó la etiología hipertensiva $(47,2 \%)$ y la isquémica $(32,8 \%)$. Los pacientes con Stevenson C en su mayoría tenían etiología isquémica (40\%), seguida por etiología hipertensiva (35\%) y causa por arritmias (25\%). En el grupo de pacientes clasificados como Stevenson L, las principales etiologías de falla cardiaca fueron igualmente la hipertensiva e isquémica.

El factor desencadenante más frecuente de insuficiencia cardiaca fue la presencia de taquiarritmias o bradiarritmias $(17,5 \%)$, seguido por infecciones $(17,2 \%)$ y exacerbación de neumopatía crónica $(16,3 \%)$. La no adherencia al tratamiento médico ambulatorio contribuyó a la descompensación en el $13,9 \%$ del total de casos, pero aumentó a $19,2 \%$ en aquellos con falla cardiaca conocida $(n=261)$. Otras causas menos frecuentes fueron la disfunción renal aguda $(8,6 \%)$, síndrome coronario agudo $(7,2 \%)$, anemia $(3,9 \%)$ y diabetes descompensada (3,6\%). En el $19,9 \%$ de los individuos analizados hubo dos o más factores desencadenantes concomitantes. En el $15,2 \%$ de casos no se encontró causa clara de descompensación.

En pacientes con FEVI reducida, el principal factor desencadenante fue también la presencia de arritmias cardíacas, 
$(19,7 \%)$, seguida por la no adherencia al tratamiento ambulatorio $(18,6 \%)$ y las infecciones $(14,7 \%)$. En aquellos con FEVI preservada, los factores desencadenantes más comunes fueron la infección $(20,4 \%)$, la exacerbación de neumopatía crónica (18,6\%) y las arritmias (16,2\%).

En relación con la clasificación Stevenson al ingreso, aquellos clasificados como Stevenson B tuvieron como principal factor desencadenante las arritmias cardíacas (17,3\%), seguido por las infecciones y la exacerbación de la neumopatía de base $(16,7 \%$ y $16,4 \%$ respectivamente). El total de individuos con disfunción renal como causa de la agudización de la falla cardiaca fueron clasificados como Stevenson B. En pacientes Stevenson C al ingreso, el principal factor desencadenante fue la infección (25\%), seguido por arritmias (20\%); en el $15 \%$ de casos hubo descompensación relacionada con la neumopatía de base y no adherencia al tratamiento ambulatorio. El 25\% tenía dos o más factores desencadenantes. En pacientes clasificados como Stevenson $L$ el principal factor desencadenante fue la hipertensión arterial no controlada $(33,3 \%)$.

\section{Estancia hospitalaria y mortalidad}

La tabla 5 resume los aspectos más importantes de estancia hospitalaria y mortalidad. La mediana de estancia hospitalaria fue de 6 días (RIC 4-9 días), sin diferencias significativas entre los pacientes con fracción de eyección reducida y preservada, pero con una mediana de estancia hospitalaria más prolongada en el subgrupo con FEVI intermedia ( 9 días, RIC 5-11 días). La mediana de estancia hospitalaria fue discretamente más prolongada en el grupo Stevenson $C$ ( 9 días, RIC 7-10 días).

Solo 18 pacientes $(4,5 \%)$ requirieron hospitalización en unidad de cuidados intensivos $(\mathrm{UCl})$, similar en los pacientes con FEVI preservada $(5,4 \%)$ y reducida $(4,4 \%)$, pero mayor en individuos con FEVI intermedia (15,8\%). 12 de los 336 pacientes clasificados como Stevenson B (3,6\%), 3 de los 20 individuos del grupo Stevenson C (15\%) y 3 de los 6 sujetos con clasificación Stevenson L (50\%) requirieron estancia en UCl. La mediana de estancia en UCI fue similar en todos los subgrupos (4 días, RIC 2-6 días).

En total 30 pacientes fallecieron $(8,3 \%)$ : 12 con FEVI preservada $(7,2 \%$ del total con $\mathrm{FEVI}>40 \%)$ y 11 individuos con $\mathrm{FEVI}$ reducida (6\% del total con $\mathrm{FEVI}<40 \%$ ). La mortalidad fue mayor en sujetos con FEVI intermedia que en aquellos con $\mathrm{FEVI}>50 \%$ ( $21,1 \%$ y $3,4 \%$ respectivamente). La mortalidad también fue mayor en el grupo con FEVI desconocida (3 de 11 pacientes, 27,3\% de mortalidad). Algunas características clínicas en los 30 individuos fallecidos fueron diferentes al grupo de sujetos que no fallecieron durante la hospitalización: mediana de edad mayor (81 vs. 75 años, respectivamente), mayor cantidad de pacientes con descompensaciones previas conocidas $(53,3 \%$ vs. $39,7 \%$ de los sujetos), menor número de pacientes con clasificación Stevenson $B(66,7 \%$ vs. $95,2 \%)$ y mayor número de pacientes con FEVI intermedia (15,4\% vs. 4,6\%). Las causas de mortalidad fueron las siguientes: 15 casos por choque cardiogénico, 7 casos por choque séptico, 7 casos de falla ventilatoria (5 sujetos con neumonía, 1 episodio de hemoptisis masiva y 1 evento de neumotórax espontáneo de causa no determinada) y 1 caso de arritmia secundaria a hiperpotasemia.
Los individuos con infección como factor desencadenante de descompensación de la insuficiencia cardiaca constituyeron el grupo con mayor mortalidad (50\% de fallecidos), seguido por síndrome coronario agudo (20\%), arritmia y exacerbación de neumopatía (13,3\% cada uno). El $26,7 \%$ de los sujetos fallecidos tuvo dos o más factores desencadenantes de agudización de insuficiencia cardiaca.

\section{Discusión}

En este estudio se describen pacientes con insuficiencia cardiaca aguda admitidos a un hospital universitario de cuarto nivel de complejidad, encontrando similitudes con las cohortes internacionales ${ }^{1-3}$ : mediana de edad de 76 años, predominio del género femenino, antecedente conocido de falla cardiaca en 3 de cada 4 pacientes ingresados y alta carga de comorbilidades. Las diferencias más destacadas fueron menor frecuencia de pacientes con cardiopatía isquémica y mayor tasa de mortalidad.

La disnea como síntoma cardinal de falla cardiaca fue el más reportado en la cohorte $(90 \%)$ y coincide con registros internacionales, en los cuales entre el 65 y el $87 \%$ ingresan a urgencias por disnea y deterioro de su clase funcional. Es infrecuente no encontrar este síntoma en un paciente con insuficiencia cardiaca aguda, aunque su presencia es inespecífica y no descarta otros diagnósticos diferenciales.

La no adherencia farmacológica representa un factor desencadenante potencialmente modificable o evitable. Registros prospectivos latinoamericanos como el ICARO, BREATHE y CONAREC XVIII, evidenciaron que la no adherencia farmacológica explica entre el $13 \%$ y el $29 \%$ de descompensaciones de falla cardiaca ${ }^{13-15}$. En nuestra cohorte el $19,2 \%$ de pacientes con falla cardiaca conocida presentaron descompensación por adherencia inadecuada al tratamiento farmacológico, pero este número pudo ser incluso mayor, considerando la baja adherencia (cercana al 35\%) a terapia farmacológica como betabloqueantes, IECA o ARA II en aquellos con indicación clara (FEVI reducida o cardiopatía isquémica). Infortunadamente el carácter retrospectivo del estudio limita el análisis a profundidad de este aspecto. Se deben hacer mayores esfuerzos en la educación de estos pacientes, garantizar la entrega oportuna de medicamentos y capacitar al talento humano en salud para mejorar el tratamiento global de la falla cardiaca, impactando en causas prevenibles de descompensación.

La cardiopatía isquémica solo representó el 33\% del total de casos de falla cardiaca, en contraposición con la asociación clásica de enfermedad coronaria como evento disparador de falla cardiaca en cerca del $70 \%$ de $\operatorname{casos}^{16}$, pero concuerda con la epidemiología descrita en el estudio Inter-CHF, en el que la cardiopatía isquémica solo explicó el 1 de cada 4 casos de falla cardiaca en la población latinoamericana ${ }^{17}$. Sin embargo, es importante destacar que la enfermedad isquémica se ha posicionado en los últimos años como la primera causa de muerte en Colombia, con tasas de mortalidad que superan los 68 por 100.000 habitantes $^{18}$. Con el mejoramiento continuo en las estrategias de detección, diagnóstico y manejo de la población con enfermedad coronaria, se espera que el impacto de la cardiopatía isquémica en nuestra población con falla cardiaca sea más notorio en los próximos años. La hipertensión 
Tabla 5 Estancia hospitalaria y mortalidad

\begin{tabular}{|c|c|c|c|c|c|}
\hline Variable & Total & $\begin{array}{l}\text { Días de estancia } \\
\text { hospitalaria }\end{array}$ & $\begin{array}{l}\text { Requerimiento } \\
\text { de } \mathrm{UCl}\end{array}$ & $\begin{array}{l}\text { Días estancia } \\
\text { en UCl }\end{array}$ & $\begin{array}{l}\text { Mortalidad } \\
\text { intrahospitalaria }\end{array}$ \\
\hline $\begin{array}{l}\text { Total } \\
\text { Según la fracción } \\
\text { de eyección }\end{array}$ & 361 (100\%) & $6(4-9)$ & $18(5 \%)$ & $4(2-6)$ & $30(8,3 \%)$ \\
\hline $\begin{array}{l}\text { FEVI menor del } \\
40 \%\end{array}$ & $183(50,7 \%)$ & $6(4-9)$ & $8(4,4 \%)$ & $4(2-6)$ & $14(7,7 \%)$ \\
\hline $\begin{array}{l}\text { FEVI mayor del } \\
40 \%\end{array}$ & $167(46,3 \%)$ & $6(4-10)$ & $9(5,4 \%)$ & $4(2-7)$ & $12(7,2 \%)$ \\
\hline $\begin{array}{l}\text { FEVI } 41-50 \% \\
\text { Según la } \\
\text { clasificación de } \\
\text { Stevenson }\end{array}$ & $19(5,3 \%)$ & $9(5-11)$ & $3(15,8 \%)$ & $4(4-8)$ & $4(21,1 \%)$ \\
\hline$B$ & $335(92,8 \%)$ & $6(4-9)$ & $12(3,6 \%)$ & $4(2-5)$ & $20(6 \%)$ \\
\hline C & $20(5,5 \%)$ & $9(7-10)$ & $3(15 \%)$ & $4(4-7)$ & 7 (35\%) \\
\hline$L$ & $6(1,7 \%)$ & $6(3-11)$ & 3 (50\%) & $1(0-8)$ & $3(50 \%)$ \\
\hline
\end{tabular}

arterial interviene como causa, asociada a los episodios de descompensación y como comorbilidad. En nuestro grupo de pacientes, estuvo presente en cerca del $80 \%$ de individuos, y se vinculó como la primera causa de falla cardiaca $(46,3 \%$ de casos).

Sobresale la alta frecuencia de comorbilidades descompensadas asociadas a la insuficiencia cardiaca aguda. También destaca la presencia de infección, que se asoció a cerca de 1 en cada 5 sujetos, al igual que la presencia de dos o más causas de descompensación en un mismo individuo. Fueron diversas las causas de descompensación descritas, sin un claro predominio de alguna de ellas (tabla 4). Lo anterior refleja la complejidad del paciente con falla cardiaca, la necesidad de un enfoque integral, así como de una evaluación sistemática y exhaustiva que permita identificar y tratar de manera adecuada las múltiples causas de agudización. El tratamiento acertado de las patologías de base puede disminuir sustancialmente las complicaciones que acarrea la insuficiencia cardíaca aguda y disminuir directamente la tasa de reingresos hospitalarios por descompensación.

La mortalidad intrahospitalaria fue similar a la descrita recientemente en una cohorte bogotana ${ }^{11}$, pero sustancialmente mayor que lo reportado en otras cohortes nacionales e internacionales ${ }^{1-4,7,10,15}$. Debe tenerse en cuenta que los grandes registros internacionales fueron diseñados con el objetivo de mejorar las medidas de desempeño hospitalario y son el reflejo de una aplicación adecuada de tratamiento basados en la evidencia. El 50\% de los pacientes del estudio falleció por infección asociada a la descompensación cardiaca, la cual por sí misma supone mayor riesgo de mortalidad $^{19}$. También deben tenerse en cuenta factores sociales, biológicos, étnicos y del desarrollo que pueden impactar en desenlaces clínicos. La mayor edad, mayor carga de comorbilidades, mayor presencia de sujetos con FEVI reducida y mayor proporción de pacientes con cardiopatía isquémica en los pacientes de nuestra cohorte explican la diferencia en mortalidad con otros estudios del ámbito nacional ${ }^{7,10}$.

El grupo de pacientes con fracción de eyección intermedia representó únicamente el $5 \%$ de toda la muestra, pero tuvo algunas características que sugieren un comportamiento diferente: mediana de edad mayor (81 vs. 76 años), al igual que la estancia hospitalaria (9 vs. 6 días), mayor proporción de sujetos en $\mathrm{UCl}(15,8 \%$ vs. $5 \%)$ y mortalidad considerablemente mayor $(21,1 \%$ vs- $8,9 \%)$. Por la escasa cantidad de pacientes y las características del estudio no fue posible establecer otras particularidades de este subgrupo de pacientes, pero se precisa la necesidad de caracterizar clínica y epidemiológicamente a este subgrupo de individuos ${ }^{20}$.

El estudio proporciona información acerca de la epidemiología local de un hospital de cuarto nivel de la ciudad de Medellín; permitió determinar diferencias según la fracción de eyección, caracterizar un subgrupo con FEVI intermedia, evaluar diferencias según el perfil hemodinámico de presentación y estancia en $\mathrm{UCl}$, uso de inotrópicos, vasopresores y mortalidad intrahospitalaria. Fue posible la caracterización de factores desencadenantes y de etiología, con utilidad real en la práctica clínica.

En cuanto a las limitaciones del estudio, se llevó a cabo en un único centro representando un posible sesgo de referencia que no demuestre necesariamente la realidad global de la falla cardiaca aguda en Colombia. Fue retrospectivo, lo que limitó el acceso a información, recolección y análisis de datos, especialmente en aquellos registros donde fue necesario inferir y tácitamente adquirir la información. El seguimiento fue a corto plazo y se limitó a mortalidad intrahospitalaria. Por la escasa cantidad de muestras de péptido natriurético cerebral hechas en la institución, no fue posible correlacionar el diagnóstico de insuficiencia cardiaca aguda con este examen de laboratorio, el cual es una herramienta básica para el estudio y pronóstico de los pacientes que consultan al servicio de urgencias con diagnóstico presuntivo de falla cardiaca.

\section{Conclusiones}

La población con insuficiencia cardiaca aguda de esta cohorte presenta similitudes a las descritas en otras 
poblaciones internacionales y en algunas nacionales, pero con mortalidad mayor, asociada principalmente a infección concomitante y a síndrome coronario agudo. Se realizó una caracterización de factores desencadenantes y de etiología, con utilidad en la práctica clínica. Hubo una alta carga de comorbilidades, cuya descompensación impactó de manera significativa en la agudización de la falla cardiaca. El subgrupo con fracción de eyección intermedia presentó características que sugieren un comportamiento diferente y ameritan caracterizar con mayor detalle.

\section{Financiación}

El estudio fue financiado en su totalidad por los autores.

\section{Conflicto de intereses}

Ninguno.

\section{Agradecimientos}

Al doctor Fabián Jaimes Barragán, internista epidemiólogo, por su asesoría en aspectos epidemiológicos durante el desarrollo de la investigación y redacción del manuscrito.

A la doctora Clara Inés Saldarriaga, internista cardióloga, por su asesoría y aportes en la revisión del manuscrito.

\section{Bibliografía}

1. Cleland JGF, Swedberg K, Follath F, Komajda M, CohenSolal A, Aguilar JC, et al. The EuroHeart Failure survey programme- a survey on the quality of care among patients with heart failure in Europe. Part 1: patient characteristics and diagnosis. Eur Heart J [Internet]. 2003 Mar;24:442-63 [Acceso 2017 May 21], Disponible en: http://www.ncbi.nlm.nih.gov/pubmed/12633546.

2. Adams KF, Fonarow GC, Emerman CL, LeJemtel TH, Costanzo MR, Abraham WT, et al. Characteristics and outcomes of patients hospitalized for heart failure in the United States: Rationale, design, and preliminary observations from the first 100,000 cases in the Acute Decompensated Heart Failure National Registry (ADHERE). Am Heart J. 2005;149:209-16.

3. Fonarow GC1; ADHERE Scientific Advisory Committee. The Acute Decompensated Heart Failure National Registry (ADHERE): opportunities to improve care of patients hospitalized with acute decompensated heart failure. Rev Cardiovasc Med. 2003;4 Suppl 7:21-30.

4. Stewart S, Demers C, Murdoch DR, McIntyre K, MacLeod ME, Kendrick $S$, et al. Substantial between-hospital variation in outcome following first emergency admission for heart failure. Eur Heart J [Internet]. 2002 Apr;23:650-7 [Acceso 2017 May 21]Disponible en: https://academic.oup.com/eurheartj/article-lookup/doi/ 10.1053/euhj.2001.2890.

5. Teerlink GMF JR. Diagnosis and Management of Acute Heart Failure. In: Braunwald's heart disease: a textbook of cardiovascular medicine. $10^{\text {th }}$. ed Philadelfia: Elsevier, Saunders; 2015. p. 484-511.
6. Farmakis D, Parissis J, Lekakis J. Insuficiencia cardiaca aguda: epidemiologı ń y prevencio. 2015;68:245-8.

7. Lancheros A, Valencia Y, Chaves W. Insuficiencia cardiaca aguda: Factores asociados con mortalidad. 2009;18:166-74.

8. Castaño Castrillón J, Giraldo F, Herrera D, Jiménez JJ, Noreñavillada JM, Restrepo $M$, et al. Characterization of patients with cardiac insufficiency in the first level of attention in Manizales (Colombia). Arch Med. 2010;10:127-38.

9. Ospina Serrano AV, Gamarra Hernandez G. Características clínicas y epidemiológicas de la insuficiencia cardiaca en el Hospital Universitario Ramón Gonzalez Valencia de Bucaramanga. Colombia. Rev SALUD UIS; Rev Salud UIS. 2010;36:125-31.

10. Senior J, Saldarriaga C, Rendón J. Descripción clínicoepidemiológica de los pacientes con falla cardiaca aguda que consultan al servicio de urgencias. Acta Medica Colomb. 2011;36:125-9.

11. Chaves WG, Diaztagle JJ, Sprockel JJ, Hernández JI, Benavidez JM, Henao DC, et al. Factors associated with mortality in patients with decompensated heart failure. Acta Medica Colomb. Acta Médica Colombiana;. 2014;39:314-20.

12. McKee PA, Castelli WP, McNamara PM, Kannel WB. The Natural History of Congestive Heart Failure: The Framingham Study. N Engl J Med [Internet]. 1971 Dec 23;285:1441-6 [Acceso 2017 Jul 30], Disponible en: http://www.ncbi.nlm.nih.gov/pubmed/5122894.

13. Albuquerque DCde, Souza Neto JDde, Bacal F, Rohde LEP, Bernardez-Pereira S, Berwanger O, et al. I Brazilian Registry of Heart Failure - Clinical Aspects. Care Quality and Hospitalization Outcomes. Arq Bras Cardiol [Internet]. 2015. Disponible en: http://www.gnresearch.org/doi/10.5935/abc.20150031.

14. Corradi L, Pérez G, Costabel JP, González N, Da Rosa W, Amirano MA, et al. XVIII conarec registry on decompensated heart failure in Argentina. Rev Argent Cardiol [Internet]. 2014;82:494-503. Disponible en: http://www.embase.com/search/results? subaction=viewrecord\&from=export\&id= L604655836\%5Cnhttps: / / doi.org/10.7775/rac.v82.i6.4848.

15. Castro GP, Vukasovic RJL, Garcés SE, Sepúlveda ML, Ferrada KM, Alvarado OS. Insuficiencia cardíaca en hospitales chilenos: Resultados del Registro Nacional de Insuficiencia Cardíaca. Grupo ICARO. Rev Med Chil. 2004;132:655-62.

16. Senior JM. Guía de Práctica Clínica para la prevención, diagnóstico, tratamiento y rehabilitación de la falla cardíaca en población mayor de 18 años clasificación B, C y D. Bogotá: Ministerio de Salud y Proteccion Social; 2015. p. 1-38.

17. Dokainish H, Teo K, Zhu J, Roy A, Alhabib KF, Elsayed A, et al. Heart Failure in Africa, Asia, the Middle East and South America: The INTER-CHF study. Int J Cardiol [Internet]. Elsevier Ireland Ltd. 2016;204:133-41. Disponible en: https://doi.org/10.1016/j.ijcard.2015.11.183.

18. Senior JM, Lugo LH, Acosta N, Acosta JL, Díaz J, Osío OH, et al. Guía de práctica clínica para El Síndrome Coronario Agudo. 2013:1-255.

19. Rhodes A, Evans LE, Alhazzani W, Levy MM, Antonelli M, Ferrer R, et al. Surviving Sepsis Campaign [Internet]. Critical Care Medicine. 2017;45:486-552. Disponible en: http: / / insights.ovid.com/crossref?an=00003246-20170300000015.

20. Ponikowski P, Voors AA, Anker SD, Bueno H, Cleland JGF, Coats AJS, et al. 2016 ESC Guidelines for the diagnosis and treatment of acute and chronic heart failure. Eur Heart J. 2016;37:2129-200. 